\title{
Genome sequence of type strain of Staphylococcus aureus subsp. aureus
}

Bong-Soo Kim ${ }^{1}$, Hana $\mathrm{Yi}^{2,3,4}$, Jongsik Chun ${ }^{1,5}$ and Chang-Jun Cha ${ }^{6^{*}}$

\begin{abstract}
Background: Staphylococcus aureus is a pathogen that causes food poisoning and community-associated infection with antibiotic resistance. This species is an indigenous intestinal microbe found in infants and not found in adult intestine. The relatively small genome size and rapid evolution of antibiotic resistance genes in the species have been drawing an increasing attention in public health. To extend our understanding of the species and use the genome data for comparative genomic studies, we sequenced the type strain of S. aureus subsp. aureus DSM $20231^{\top}$.

Results: Seventeen contigs were generated using hybrid assembly of sequences derived from the Roche 454 and Illumina systems. The length of the genome sequence was 2,902,619 bases with a $\mathrm{G}+\mathrm{C}$ content of $32.8 \%$. Among the 2,550 annotated CDSs, 44 CDSs were annotated to antibiotic resistance genes and 13 CDSs were related to methicillin resistance. It is interesting to note that this strain was first isolated in 1884 before methicillin was generally used on patients.

Conclusions: The present study analyzed the genome sequence of $S$. aureus subsp. aureus type strain as the reference sequence for comparative genomic analyses of clinical isolates. Methicillin resistance genes found in the genome indicate the presence of antibiotic resistance mechanism prior to the usage of antibiotics. Further comparative genomic studies of methicillin-resistant strains based on this reference genome would help to understand the evolution of resistance mechanism and dissemination of resistance genes.
\end{abstract}

Keywords: Staphylococcus aureus subsp. aureus, Genome sequencing, Type strain, Hybrid assembly

\section{Background}

Staphylococcus aureus is a member of normal microbiota in human body and also known as an opportunistic pathogen. This species can cause a broad range of nosocomial and community-associated infections, and the antibiotic resistance of the species has been studied for many years [1]. S aureus was also reported as the predominant species in infant feces, and decreased toward adulthood due to the colonization of complex gut microbiota [2,3]. The species can spread through skin-to-skin contact with colonized individuals, and cause a global epidemic as antibiotic resistant strains [4]. Foodborne illness can be caused by enterotoxin-producing $S$. aureus with symptoms such as diarrhea, nausea and abdominal cramps [5]. Recently, S. aureus was detected in irritable bowel syndrome (IBS) subjects [6].

\footnotetext{
*Correspondence: cjcha@cau.ac.kr

${ }^{6}$ Department of Systems Biotechnology, Chung-Ang University, Anseong, Republic of Korea

Full list of author information is available at the end of the article
}

Many strains of S. aureus subsp. aureus were genomesequenced and submitted to public databases due to the importance in antibiotic resistance and the possibility of nosocomial infections even in health care and community settings [7-9]. However, type strain of this species has not been genome-sequenced yet. Type strain is usually the firstly isolated strain of the species, and exhibits all of the relevant phenotypic and genotypic properties cited in the species circumscriptions. Therefore, the genome sequence of type strain is important to analyze the phenotypic and genotypic characteristics of species. In the present study, we analyzed the whole genome sequence of $S$. aureus subsp. aureus type strain as the standard reference genome required for $S$. aureus studies.

\section{Methods}

\section{Strain information}

Type strain of S. aureus subsp. aureus (DSM 20231 ${ }^{\mathrm{T}}$ ) was obtained from Deutsche Sammlung von Mikrooganismen und Zellkulturen GmbH (DSMZ; Barunschweig, Germany). 
The strain was known to be non-motile, non-sporeforming, Gram-positive cocci (0.5-1.0 $\mu \mathrm{m}$ in diameter), facultatively anaerobic and producing enterotoxin. Optimal growth is observed at $30-37^{\circ} \mathrm{C}$ on trypticase soy yeast extract media containing $10 \% \mathrm{NaCl}$ [10].

\section{Genomic DNA extraction}

Genomic DNA was extracted using a Wizard Genomic DNA Isolation kit (Promega, Madison, WI, USA). The concentration of extracted DNA was quantified using a PicoGreen dsDNA Assay kit (Invitrogen, Carlsbad, CA, USA), and the contamination of DNA or cultured strain was checked by sequencing the $16 \mathrm{~S}$ rRNA gene using the ABI 3730 DNA sequencing machine (Applied Biosystems, Foster City, CA, USA).

\section{Whole genome sequencing}

The draft genome sequence of strain DSM $20231^{\mathrm{T}}$ was determined by a combination of Illumina Genome Analyzer IIx (150 bp paired end) and Roche 454 (8-kb insert paired end) sequencing systems. The sequencing library was prepared with the TruSeq DNA LT Sample Prep kit (Illumina, San Diego, CA, USA) for the Illumina system, and the library for the Roche 454 system was prepared using the GS FLX Titanium Rapid Library Preparation kit (Roche Diagnostics, Branford, CT, USA).

\section{Assembly and annotation of genome sequence}

Sequencing reads obtained from the Illumina system were assembled using the CLC genomic workbench 5.5 (CLC Bio, Denmark), and the reads obtained from the Roche 454 sequencing system were assembled using the GS Assembler 2.6 (Roche Diagnostics). The assembled contigs from each sequencing system were corrected in their order using the published reference genomes. Hybrid assembly of contigs generated by both systems was conducted using the CodonCode Aligner (CodonCode Co. MA, USA). In brief, the contigs generated by each sequencing system were reassembled together using the CodonCode Aligner. Reassembly of hybrid contigs and unassembled contigs were repeated until the number of hybrid contigs did not change. Contigs of short length ( $<500$ bp) were removed from the hybrid result file. Prediction of genes was performed using Glimmer 3 [11], and annotation was conducted by homology search against the Clusters of Orthologous Groups (COG)

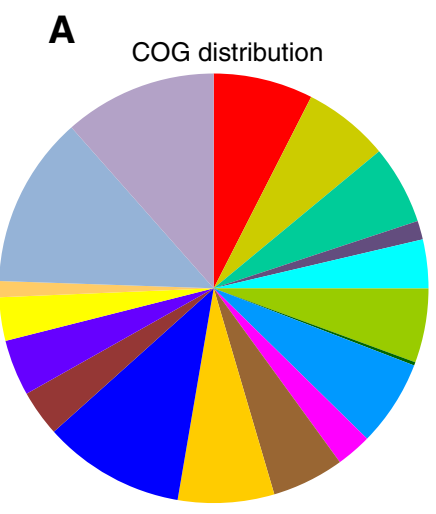

B

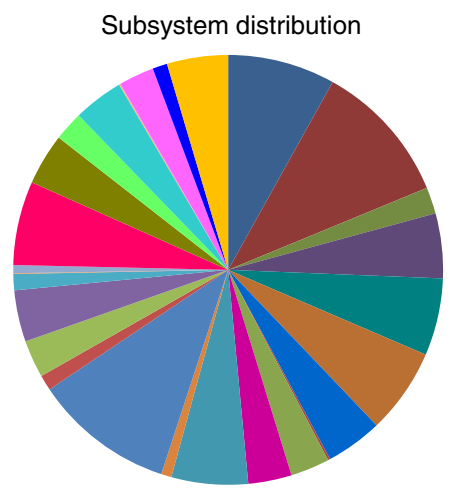

COG category feature (counts)

Translation, ribosomal structure and biogenesis (149)

Transcription (129)

Replication, recombination and repair (118)

- Cell cycle control, cell division, chromosome partitioning (28)

Posttranslational modification, protein turnover, chaperones (73)

Cell wall/ membrane/ envelope biogenesis (111)

Cell motility (5)

Inorganic ion transport and metabolism (129)

- Signal transduction mechanisms (53)

Energy production and conversion (108)

Carbohydrate transport and metabolism (144)

- Amino acid transport and metabolism (212)

Nucleotide transport and metabolism (69)

Coenzyme transport and metabolism (84)

Lipid transport and metabolism (65)

Secondary metabolites biosynthesis, transport and catabolism (24)

General function prediction only (257)

Function unknown (229)

Subsystem feature (counts)

Amino Acids and Derivatives (146)

Carbohydrates (193)

Cell Division and Cell Cycle (36)

Cell Wall and Capsule (88)

Clustering-based subsystems (105)

Cofactors, Vitamins, Prosthetic Groups, Pigments (116)

DNA Metabolism (77)

DNA Metabolism (77)

Fatty Acids, Lipids, and Isoprenoids (53)

Fatty Acids, Lipids, and Isoprenoids
Iron acquisition and metabolism (59)

- Iron acquisition and metabolisn

Membrane Transport (104)
Metabolism of Aromatic Compounds (14)

Miscellaneous (191)

Nitrogen Metabolism (21)

Nucleosides and Nucleotides (51)

Phages, Prophages, Transposable elements, Plasmids (70)

Phosphorus Metabolism (22)

Photosynthesis (1)

Potassium metabolism (11)

Protein metabolism (114)

Regulation and Cell signaling (146)

Respiration (40)

RNA Metabolism (68)

Secondary Metabolism (2)

Stress Response (48)

Sulfur Metabolism (48)

Virulence, Disease and Defense (83)

Figure 1 Statistics of annotated genes for Staphylococcus aureus subsp. aureus DSM $20231^{\top}$ based on COG (A) and SEED (B) databases. 
and SEED database [12,13]. Prediction of multilocus sequencing typing (MLST) was performed using assembled contigs [14].

\section{Comparative genomics}

A total of 441 genome sequences of strains that belong to $S$. aureus subsp. aureus were obtained from EzGenome database (http://ezgenome.ezbiocloud.net), and used to calculate average nucleotide identity (ANI) values [15] with strain DSM $20231^{\mathrm{T}}$. For ANI calculation, the query genome was cut into small fragments (1020 bp), and highscoring pairs between two genome sequences were selected by BLAST algorithm [16]. Then, a dendrogram was constructed using calculated ANI values by the unweighted pair group method.

Five genome sequences (an ANI value of $>99.78 \%$ with strain DSM $20231^{\mathrm{T}}$ ) were selected as the closest genomes and compared with strain DSM $20231^{\mathrm{T}}$ by using comparative genomic method as described previously [17]. Briefly, homologous regions in a target genome to query ORFs were determined using the BLASTN program, and aligned using a pairwise global alignment. The matched region in the subject contig was extracted and saved as a homolog.

\section{Quality assurance}

A potential contamination was evaluated by identification of 16S rRNA gene amplified from extracted DNA before the whole genome sequencing and by comparison of $16 \mathrm{~S}$ rRNA gene in draft genome after sequencing. 16S rRNA genes in assembled contigs were found using the rRNA selector [18] and identified using the EzTaxon-e database [19]. Bioinformatic assembly was checked by a comparison of the obtained genome sequence with published genomes of the same species using ANI values [15].

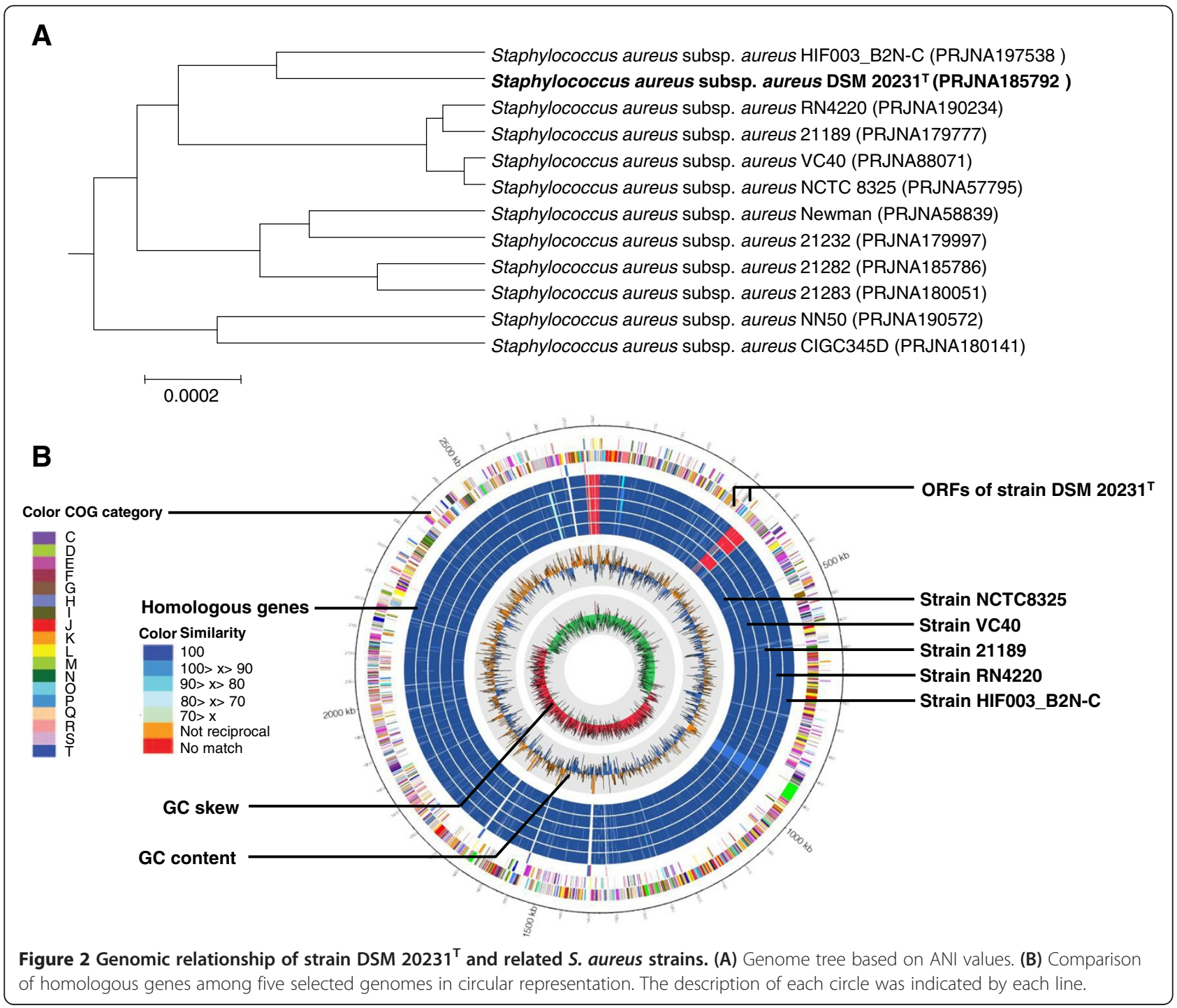




\section{Initial findings}

A total of 17 contigs $(\mathrm{N} 50=313,118 \mathrm{bp})$ were generated from a hybrid assembly of reads from Illumina (6,413,077 reads of 150 bp paired end; > 350-fold coverage) and Roche 454 (240,863 reads of $8 \mathrm{~Kb}$-insert paired end; > 14fold coverage) systems. The genome size of strain DSM $20231^{\mathrm{T}}$ was $2,761,522$ bases with $32.8 \% \mathrm{G}+\mathrm{C}$ content. The genome contained 2,550 predicted protein-coding sequences (CDSs), 57 tRNA genes and 12 rRNA genes. Results of the genome annotation are shown in Figure 1. For the COG distribution, $\mathrm{R}$ (General function prediction only; 257 ORFs), S (Function unknown; 211 ORFs), and E (Amino acid transport and metabolism; 212 ORFs) were abundant categories (over 10\% of total COG matched counts). Genes responsible for carbohydrates (193 ORFs), miscellaneous (191 ORFs), amino acid metabolism (146 ORFs) and cell signaling (146 ORFs) were abundant among the SEED subsystem categories.

The genome tree of $S$. aureus strains was constructed by using ANI calculation (Figure 2A), and strains HIF003B2N-C, RN4220, 21189, VC40, and NCTC 8325 were chosen based on ANI values for the comparative analysis. Strain HIF003_B2N-C was recovered as the closest genome of the sequenced genome in the genome tree. The number of different gene contents between strains of DSM $20231^{\mathrm{T}}$ and HIF003-B2N-C was 35 ORFs, and the highest different number between them was observed in $\mathrm{K}$ (Transcription) and $\mathrm{L}$ (Replication, recombination and repair) categories. Genome sequences among selected strains for comparison were similar to each other, and most of the different ORFs were hypothetical proteins, replication-associated proteins, and transposases. Comparison of homologous genes among the selected genomes is given in Figure 2B.

In subsystem distribution of the sequenced genome, 83 genes (4.6\% of total subsystem counts) were annotated to virulence, disease and defense category, and $91.6 \%$ of genes (76 ORFs) in this category were annotated to be responsible for adhesion and antibiotic resistance. Adhesion to human intestinal mucus and antibiotic resistance of S. aureus are important characteristics of pathogens. The highest number of predicted protein sequences (13 CDSs) among 44 CDSs in antibiotic resistance subcategory was annotated to methicillin resistance-related genes (Table 1). In the case of the five related genomes, the numbers of these genes were much smaller than that in the type strain (3 CDSs in HIF003_B2N-C and RN4220, 2 CDSs in NCTC 8325, 1 CDS in VC40, and no hit in strain 21189). The methicillin resistance of $S$. aureus was first reported in 1961 [20]. However, strain DSM $20231^{\mathrm{T}}$ was isolated in 1884 from human pleural fluid [21]. This implies that $S$. aureus had already possessed potential genes for methicillin resistance before methicillin was introduced in 1960. Our finding can provide an insight into history and evolution of methicillin resistance. The predicted MLST of strain DSM $20231^{\mathrm{T}}$ and the five selected strains for comparison were all ST8 in the clonal complex (CC) 8, where the first MRSA clinical isolate is ST250 [1]. In addition, genes related to arsenic resistance, fluoroquinolone resistance, fosfomycin resistance, vancomycin resistance, multidrug resistance efflux pumps, cobaltzinc-cadmium resistance, and copper homeostasis were annotated in the genome sequence of strain DSM $20231^{\mathrm{T}}$. The presence of antibiotic resistance genes in the genome of this strain implies that antibiotic resistances of this species have evolved for a long time before synthetic antibiotics were used.

\section{Future directions}

The genome sequence of $S$. aureus subsp. aureus type strain can be used as a standard reference genome

Table 1 Summary of CDSs annotated to methicillin resistance

\begin{tabular}{|c|c|c|c|}
\hline Contig number & Length (bp) & Seed subsystem & Seed function \\
\hline 2 & 1,194 & Methicillin_resistance_in_Staphylococci & FmtA protein involved in methicillin resistance \\
\hline 2 & 1,245 & Methicillin_resistance_in_Staphylococci & FmhC protein of FemAB family \\
\hline 2 & 369 & Methicillin_resistance_in_Staphylococci & FemC factor involved in methicillin resistance \\
\hline 2 & 2,523 & Methicillin_resistance_in_Staphylococci & FmtC (MrpF) protein involved in methicillin resistance \\
\hline 2 & 1,263 & Methicillin_resistance_in_Staphylococci & FmhA protein of FemAB family \\
\hline 2 & 1,260 & Methicillin_resistance_in_Staphylococci & FmhA protein of FemAB family \\
\hline 3 & 876 & Methicillin_resistance_in_Staphylococci & LytH protein involved in methicillin resistance \\
\hline 6 & 465 & Methicillin_resistance_in_Staphylococci & HTH domain protein SA1665, binds to mecA promoter region \\
\hline 9 & 1,185 & Methicillin_resistance_in_Staphylococci & HmrA protein involved in methicillin resistance \\
\hline 9 & 7,437 & Methicillin_resistance_in_Staphylococci & $\begin{array}{l}\text { FmtB (Mrp) protein involved in methicillin resistance and } \\
\text { cell wall biosynthesis }\end{array}$ \\
\hline 11 & 1,266 & Methicillin_resistance_in_Staphylococci & tRNA-dependent lipid II-glycine ligase (FmhB) \\
\hline 12 & 1,251 & Methicillin_resistance_in_Staphylococci & FmhA protein of FemAB family \\
\hline 16 & 675 & Methicillin_resistance_in_Staphylococci & Transposase for insertion sequence-like element IS431 mec \\
\hline
\end{tabular}


sequence for studying $S$. aureus strains including MRSA. Further comparative genome analyses of $S$. aureus strains will provide differences in the genomic contents found in this species and evolutionary information on resistance developments via horizontal gene transfer and mutation. These studies will also help to understand the pathogenesis of the staphylococcal diseases for infection preventions.

\section{Availability of supporting data}

The draft genome sequence of Staphylococcus aureus subsp. aureus DSM $20231^{\mathrm{T}}$ was deposited at DDBJ/ EMBL/GenBank under the accession AMYL00000000. The version described in this paper is the first version AMYL01000000.

\section{Competing interests}

The authors declare that they have no competing interests.

\section{Authors' contributions}

JC and CC designed the study. BK and HY performed experiments. BK, HY and JC analyzed the sequencing data. BK, HY and CC contributed to the writing of manuscript. All authors read and approved the final manuscript.

\section{Acknowledgements}

This work was supported by Industrial Strategic Technology Development Program (No. 10040176) funded by the Korean Ministry of Knowledge Economy (MKE), by Basic Science Research Programs through the National Research Foundation of Korea (NRF) funded by the Ministry of Science, ICT, and Future Planning (NRF-2013R1A1A3010041) and by Korea Ministry of Environment as "The Environmental Health Action Program".

\section{Author details}

${ }^{1}$ ChunLab, Inc., Seoul, Republic of Korea. ${ }^{2}$ Division of Biosystems Biomedical Science, College of Health Science, Korea University, Seoul, Republic of Korea. ${ }^{3}$ Department of Public Health Science, Graduate School, Korea University, Seoul, Republic of Korea. ${ }^{4}$ Korea University Guro Hospital, Korea University, Seoul, Republic of Korea. ${ }^{5}$ School of Biological Sciences and Bioinformatics Institute, BIO-MAX, Seoul National University, Seoul, Republic of Korea. ${ }^{6}$ Department of Systems Biotechnology, Chung-Ang University, Anseong, Republic of Korea.

Received: 28 January 2014 Accepted: 11 March 2014 Published: 17 March 2014

\section{References}

1. Chambers HF, Deleo FR: Waves of resistance: Staphylococcus aureus in the antibiotic era. Nat Rev Microbiol 2009, 7:629-641.

2. Lindberg E, Nowrouzian F, Adlerberth I, Wold AE: Long-time persistence of superantigen-producing Staphylococcus aureus strains in the intestinal microflora of healthy infants. Pediatr Res 2000, 48:741-747.

3. Bjorksten B, Sepp E, Julge K, Voor T, Mikelsaar M: Allergy development and the intestinal microflora during the first year of life. J Allergy Clin Immunol 2001, 108:516-520

4. Muto CA, Jernigan JA, Ostrowsky BE, Richet HM, Jarvis WR, Boyce JM, Farr BM: SHEA guideline for preventing nosocomial transmission of multidrug-resistant strains of Staphylococcus aureus and Enterococcus. Infect Cont Hosp Ep 2003, 24:362-386.

5. Tortora GJ: Staphylococcus food poisoning (Staphylococcal enterotoxicosis). In Microbiology An introduction. 1st edition. Edited by Tortora GJ, Funke BR, Case CL. San Francisco, CA, USA: Benjamin Cummings Publishing Company, Inc; 1995:616-618.

6. Rinttila T, Lyra A, Krogius-Kurikka L, Palva A: Real-time PCR analysis of enteric pathogens from fecal samples of irritable bowel syndrome subjects. Gut Pathog 2011, 3:6.

7. Kaplan SL, Hulten KG, Gonzalez BE, Hammerman WA, Lamberth L, Versalovic J, Mason EO: Three-year surveillance of community-acquired Staphylococcus aureus infections in children. Clin Infect Dis 2005, 40:1785-1791.
8. Grundmann H, Aires-De-Sousa M, Boyce J, Tiemersma E: Emergence and resurgence of meticillin-resistant Staphylococcus aureus as a public-health threat. Lancet 2006, 368:874-885.

9. Holden MTG, Feil EJ, Lindsay JA, Peacock SJ, Day NPJ, Enright MC, Foster TJ, Moore CE, Hurst L, Atkin R, Barron A, Bason N, Bentley SD, Chillingworth C, Chillingworth T, Churcher C, Clark L, Corton C, Cronin A, Doggett J, Dowd L, Feltwell T, Hance Z, Harris B, Hauser H, Holroyd S, Jagels K, James KD, Lennard N, Line A, et al: Complete genomes of two clinical Staphylococcus aureus strains: evidence for the rapid evolution of virulence and drug resistance. Proc Natl Acad Sci 2004, 101:9786-9791.

10. Vos PD, Garrity GM, Jones D, Krieg NR, Ludwig W, Rainey FA, Schleifer K, Whitman WB: The Firmicutes. In Bergey's Manual of Systematic Bacteriology. 2nd edition. New York, NY, USA: Springer; 2009:400-401.

11. Delcher AL, Bratke KA, Powers EC, Salzberg SL: Identifying bacterial genes and endosymbiont DNA with glimmer. Bioinformatics 2007, 23:673-679.

12. Tatusov RL, Koonin EV, Lipman DJ: A genomic perspective on protein families. Science 1997, 278:631-637.

13. Disz T, Akhter S, Cuevas D, Olson R, Overbeek R, Vonstein V, Stevens R, Edwards RA: Accessing the SEED genome databases via web services API: Tools for programmers. BMC Bioinforma 2010, 11:319.

14. Larsen MV, Cosentino S, Rasmussen S, Friis C, Hasman H, Marvig RL, Jelsbak L, Sicheritz-Ponten T, Ussery DW, Aarestrup FM, Lund O: Multilocus sequence typing of total-genome-sequenced bacteria. J Clin Microbiol 2012, 50:1355-1361

15. Goris J, Konstantinidis KT, Klappenbach JA, Coenye T, Vandamme P, Tiedje JM: DNA-DNA hybridization values and their relationship to whole-genome sequence similarities. Int J Syst Evol Microbiol 2007, 57:81-91.

16. Altschul SF, Madden TL, Schaffer AA, Zhang J, Zhang Z, Miller W, Lipman DJ: Gapped BLAST and PSI-BLAST: a new generation of protein database search programs. Nucleic Acids Res 1997, 25:3389-3402

17. Chun J, Grim CJ, Hasan NA, Lee JH, Choi SY, Haley BJ, Taviani E, Jeon YS, Kim DW, Lee JH, Brettin TS, Bruce DC, Challacombe JF, Detter JC, Han CS, Munk AC, Chertkov O, Meincke L, Saunders E, Walters RA, Huq A, Nair GB, Colwell RR: Comparative genomics reveals mechanism for short-term and long-term clonal transitions in pandemic Vibrio cholerae. Proc Natl Acad Sci 2009, 106:15442-15447.

18. Lee $\mathrm{JH}, \mathrm{Yi} H$, Chun J: rRNASelector: a computer program for selecting ribosomal RNA encoding sequences from metagenomic and metatranscriptomic shotgun libraries. J Microbio/ 2011, 49:689-691.

19. Kim OS, Cho YJ, Lee K, Yoon SH, Kim M, Na H, Park SC, Jeon YS, Lee JH, Yi H, Won S, Chun J: Introducing EzTaxon-e: a prokaryotic 16S rRNA gene sequence database with phylotypes that represent uncultured species. Int J Syst Evol Microbiol 2012, 62:716-721.

20. Barber M: Methicillin-resistant Staphylococci. J Clin Pathol 1961, 14:385-393.

21. Rosenbach FJ: Microorganismen bei den Wund-Infections-Krankheiten des Menschen. In Microorganismen bei den Wund-Infections-Krankheiten des Menschen. Edited by Bergmann JF. Wiesbarden, Germany; 1884:1-122.

doi:10.1186/1757-4749-6-6

Cite this article as: Kim et al:: Genome sequence of type strain of Staphylococcus aureus subsp. aureus. Gut Pathogens 2014 6:6.

\section{Submit your next manuscript to BioMed Central and take full advantage of:}

- Convenient online submission

- Thorough peer review

- No space constraints or color figure charges

- Immediate publication on acceptance

- Inclusion in PubMed, CAS, Scopus and Google Scholar

- Research which is freely available for redistribution 